\title{
Are the gates to be thrown open?
}

The Improving Access to Psychological Therapies (IAPT) programme is the largest investment ever made in primary care mental health. ${ }^{1}$ One of its intentions is to improve access to talking therapies to a wider population including vulnerable groups such as young people, older adults, and members of ethnic minority

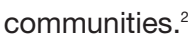

An article in this month's Journal ${ }^{3}$ reports observational data derived from the evaluations of two IAPT demonstration sites and an evaluation of psychoeducational sessions elsewhere. It compares the characteristics of patients who self-refer to IAPT with those referred from primary care, although no outcome data are provided. The authors pose the question whether a self-referral system helps improve access to psychological therapies. They conclude that such a system 'could work out extremely well and improve access for those who have not been able to get access before as well as those who have never thought of counselling'. Is this conclusion really justified?

There is no doubt that many people with high levels of mental distress are disadvantaged either due to problems of access or that their care does not meet their needs. ${ }^{4}$ Successful psychoeducational workshops have been reported to attract a wide range of selfreferred clients, including members of ethnic minority groups, with anxiety and depression scores well above average, implying appropriate referral. ${ }^{5}$ Psychoeducation workshops, however are not IAPT.

Another article ${ }^{6}$ reported no difference in symptom severity between self-referrers and GP referrals to a pilot IAPT demonstration site. In addition, those who referred themselves to the IAPT service were more closely matched to the ethnic mix of the community.

In this month's article, Brown et $a /^{3}$ acknowledge the importance of 'gatekeeping' a self-referral system and suggest the use of a self-diagnostic assessment instrument to screen self- referrals. However, they accept that one of the possible disadvantages of self-referral could be that those with relatively minor symptoms may come forward and overload the system. They also point out the danger that a predominance of articulate middle-class clients may enter the system. These are both serious considerations.

The traditional notion of 'gatekeeping' by GPs has been challenged by an increasing political focus on access to services with concomitant reduced continuity and fragmentation of GP care. ${ }^{7}$

Relationships between doctors and patients are central to both good care and good 'gatekeeping' - the skills required for the latter are the essential diagnostic ones necessary for correct and timely diagnosis with appropriate referral and subsequent management - in short, the skills to manage a biopsychosocial agenda. In addition, the identification of psychiatric and physical comorbidity (for example, thyroid disease with somatic symptoms mimicking anxiety) with a risk assessment and the subsequent coordination of care are highly likely to improve outcomes. This should also be both collaborative and comprehensive.

One immediate concern is that current depression and anxiety self-assessment tools are not validated for the purpose of lay self-diagnosis in the context of selfreferral for psychological therapies. 'Low intensity' IAPT therapists are trained to deliver defined psychological treatment packages and monitor response to and outcomes of treatment, including risk assessment. However, they are not trained in the diagnostic skills to elicit or manage a biopsychosocial agenda nor are they trained in the skills required for the exclusion of significant commonly associated comorbid conditions, such as social phobia and various personality disorders with anxiety and panic. ${ }^{8}$ Indeed IAPT therapists themselves report difficulties in managing risk and complexity as well as role confusion and difficulties in applying academic skills training to the real life world of clinical practice. ${ }^{9}$
Therefore, circumventing the current GP 'gatekeeper' system by encouraging client self-referral to IAPT therapists is likely to put them and their clients into increasingly difficult situations; ones for which they have not been trained.

The current NICE guidelines for the management of depression are based on a 'stepped care' model and correct diagnosis is crucial to deliver evidencebased psychological treatments. Extensive literature highlights the complexity of mental health diagnosis and the impact of a wide range of factors such as sex, social, and economic, as well as co-existing physical illness.

Communication skills are central to diagnosis and GPs have had good training in this area and are well placed to diagnose mental health problems and share decisions with patients about treatment options. In addition, GPs also have the necessary skills to not only manage complexity but also to understand the impact of a mental health problem on the rest of the family. Generalist care requires nothing less: the notion of replacing a holistic assessment by a GP with a selfassessment questionnaire/self-referral system to a low intensity IAPT therapist, who may have received only 3 months training before delivering their first interventions, is absurd. Not only does such a self-referral system imply that the diagnosis of depression and/or anxiety will be made by the IAPT therapist at the point of referral (with the aid of a 'gatekeeping' questionnaire) but this also runs contrary to some of the principles of the current NICE guidelines. ${ }^{10}$

The other argument in favour of selfreferral proposed by Brown et al is about improving access to psychological services by those who are traditionally more difficult to engage in services, such as members of the different minority ethnic communities. Such groups also include the younger and the older person with medically unexplained symptoms. However, IAPT therapists are not specifically trained in managing the complex problems of any of these groups 
either. ${ }^{1}$ Indeed young people are more likely to seek help for psychological problems from an informal source, such as an educational professional, than from a formal healthcare source. ${ }^{11}$ In addition, cultural competency skills are particularly important when working through the complex psychological problems that may be experienced by members of an ethnic minority community. Finally, self-referral can also reinforce the notion that depression and other mental health difficulties can only be treated by professional intervention rather than 'selfhelp' using family and community resources.

There are a number of other models to improve access being evaluated apart from self-referral; such as, the multifaceted model based on the three interlinked components of community engagement, primary care development, and sensitised psychosocial interventions. ${ }^{12}$

Illich ${ }^{13}$ in the classic Medical Nemesis, wrote about demand being created in response to the provision of services. Selfreferral to a 'free at the point of access' IAPT service fulfils many of the criteria which will generate a growing demand from the 'worried well' and the articulate middle class as IAPT is rolled out.

One of the key reasons for the existence of 'gatekeeping' in any healthcare system is to underpin demand and risk management. General practice is predicated on this and has a wide variety of demand management systems in place; for example, in the control of antibiotic prescribing, through making appointments, and secondary care referrals. Without a demand management system in place, such as that provided by the GP referral scheme, the IAPT programme, although 'bold and potentially of great importance to patients and their GPs', ${ }^{1}$ risks being overwhelmed by large numbers of 'inappropriate cases' managed and treated by relatively junior therapists.

The quality of care for patients in general practice is determined by essentials such as the good coordination and collaboration of primary healthcare team members with good communication between them, as well as being able to meet the challenges of accurate diagnosis of index and comorbid conditions within a holistic framework. Self-referral to IAPT therapists can disrupt these key processes by undermining the demand management or 'gatekeeping' systems of general practice leading to the further fragmentation of care.

\section{Nigel Mathers,}

Professor of Primary Medical Care, Academic Unit of Primary Medical Care, University of Sheffield, Sheffield.

\section{Caroline Mitchell,}

Clinical Senior Lecturer in General Practice, Academic Unit of Primary Medical Care, University of Sheffield, Sheffield.

\section{Provenance}

Commissioned; not peer reviewed.

\section{REFERENCES}

1. Byng R, Gask L. Improving access to psychological therapies: implications for mental health care in general practice. Br J Gen Pract 2009; 59(566): 640-641.

2. Improving Access to Psychological Therapies (IAPT). http://www.iapt.nhs.uk/ (accessed 31 Mar 2010).

3. Brown JSL, Boardman J, Whittinger N, Ashworth M. Can a self-referral system help improve access to psychological treatments? Br J Gen Pract 2010: 10.3399/bjgp10X501877.

4. Dowrick C. Reasons to be cheerful? Reflections on GPs' responses to depression. Br J Gen Pract 2009; DOI: 10.3399/bjgp09X454115.

5. Brown JSL, Boardman J, Elliot SA, et al. Are selfreferrers just the worried well? A cross-sectional study of self-referrers to community psycho-educational stress and self-confidence workshops. Soc Psychiatry Psychiatr Epidemiol 2005; 40: 396-401.

6. Brown JSL, Elliot SA, Boardman J, et al. Meeting the unmet need for depression services with psychoeducational self-confidence workshops: Preliminary report. Br J Psychiatry 2004; 185: 511-515.

7. Guthrie B, Saultz JW, Freeman GK, Haggerty JL. Continuity of care matters. BMJ 2008; 337: a867. DOI:10.1136/bmj.a867

8. Department of Health. Improving access to psychological therapies implementation plan: national guidelines for regional delivery. Gateway reference 9427. London: Department of Health, 2008.

9. Rizq R, Hewey M, Salvo L, et al. Reflective voices: primary care mental health workers' experiences in training and practice. Prim Health Care Res Dev 2010; 11: 72-86. DOI:10.1017/S1463423609990375.

10. National Institute for Clinical Excellence. Depression: the treatment and management of depression in adults (update). NICE Guidance CG90, 2010.

http://guidance.nice.org.uk/CG90 (accessed $12 \mathrm{Apr}$ 2010).

11. Mauerhofer A, Berchtold A, Michaud PA, Suris JC. GPs' role in the detection of psychological problems of young people: a population-based study. Br J Gen Pract 2009; DOI: 10.3399/bjgp09X454115.

12. Gask L, Rogers A, Oliver D, et al. Qualitative study of patients' perceptions of the quality of care for depression in general practice. Br J Gen Pract 2003; 53(489): 278-283

13. Illich I. Medical nemesis: the expropriation of health. London: Calder \& Boyars, 1974

DOI: 10.3399/bjgp10X484138

\section{ADDRESS FOR CORRESPONDENCE}

\section{Nigel Mathers}

Academic Unit of Primary Medical Care, University of Sheffield, Samuel Fox House, Northern General Hospital, Herries Road, Sheffield, S5 7AU.

E-mail: n.mathers@sheffield.ac.uk 